\title{
Fekete-Szegö Type Coefficient Inequalities for Certain Subclass of Analytic Functions and Their Applications Involving the Owa-Srivastava Fractional Operator
}

\author{
Serap Bulut \\ Civil Aviation College, Kocaeli University, Arslanbey Campus, 41285 Izmit, Kocaeli, Turkey \\ Correspondence should be addressed to Serap Bulut; bulutserap@yahoo.com
}

Received 7 November 2013; Accepted 28 January 2014; Published 13 March 2014

Academic Editor: Tohru Ozawa

Copyright (C) 2014 Serap Bulut. This is an open access article distributed under the Creative Commons Attribution License, which permits unrestricted use, distribution, and reproduction in any medium, provided the original work is properly cited.

A new subclass of analytic functions is introduced. For this class, firstly the Fekete-Szegö type coefficient inequalities are derived. Various known or new special cases of our results are also pointed out. Secondly some applications of our main results involving the Owa-Srivastava fractional operator are considered. Thus, as one of these applications of our result, we obtain the Fekete-Szegö type inequality for a class of normalized analytic functions, which is defined here by means of the Hadamard product (or convolution) and the Owa-Srivastava fractional operator.

\section{Introduction and Definitions}

Let $\mathscr{A}$ denote the class of functions of the form

$$
f(z)=z+\sum_{n=2}^{\infty} a_{n} z^{n}
$$

which are analytic in the unit disk

$$
\mathbb{U}=\{z \in \mathbb{C}:|z|<1\} .
$$

Also let $\delta$ denote the subclass of $\mathscr{A}$ consisting of univalent functions in $\mathbb{U}$.

Fekete and Szegö [1] proved a noticeable result that the estimate

$$
\left|a_{3}-\lambda a_{2}^{2}\right| \leq \begin{cases}-4 \lambda+3, & \lambda \leq 0 \\ 1+2 \exp \left(\frac{-2 \lambda}{1-\lambda}\right), & 0 \leq \lambda \leq 1 \\ 4 \lambda-3, & \lambda \geq 1\end{cases}
$$

holds for $f \in \mathcal{S}$. The result is sharp in the sense that for each $\lambda$ there is a function in the class under consideration for which equality holds.

The coefficient functional

$$
\phi_{\lambda}(f)=a_{3}-\lambda a_{2}^{2}=\frac{1}{6}\left(f^{\prime \prime \prime}(0)-\frac{3 \lambda}{2}\left(f^{\prime \prime}(0)\right)^{2}\right)
$$

on $f \in \mathscr{A}$ represents various geometric quantities as well as in the sense that this behaves well with respect to the rotation; namely,

$$
\phi_{\lambda}\left(e^{-i \theta} f\left(e^{i \theta} z\right)\right)=e^{2 i \theta} \phi_{\lambda}(f) \quad(\theta \in \mathbb{R}) .
$$

In fact, rather than the simplest case when

$$
\phi_{0}(f)=a_{3},
$$

we have several important ones. For example,

$$
\phi_{1}(f)=a_{3}-a_{2}^{2}
$$

represents $S_{f}(0) / 6$, where $S_{f}$ denotes the Schwarzian derivative

$$
S_{f}(z)=\left(\frac{f^{\prime \prime}(z)}{f^{\prime}(z)}\right)^{\prime}-\frac{1}{2}\left(\frac{f^{\prime \prime}(z)}{f^{\prime}(z)}\right)^{2} .
$$

Moreover, the first two nontrivial coefficients of the $n$th root transform

$$
\left(f\left(z^{n}\right)\right)^{1 / n}=z+c_{n+1} z^{n+1}+c_{2 n+1} z^{2 n+1}+\cdots
$$


of $f$ with the power series (1) are written by

$$
\begin{gathered}
c_{n+1}=\frac{a_{2}}{n}, \\
c_{2 n+1}=\frac{a_{3}}{n}+\frac{(n-1) a_{2}^{2}}{2 n^{2}},
\end{gathered}
$$

so that

$$
a_{3}-\lambda a_{2}^{2}=n\left(c_{2 n+1}-\mu c_{n+1}^{2}\right),
$$

where

$$
\mu=\lambda n+\frac{n-1}{2} .
$$

Thus it is quite natural to ask about inequalities for $\phi_{\lambda}$ corresponding to subclasses of $\mathcal{S}$. This is called FeketeSzegö problem. Actually, many authors have considered this problem for typical classes of univalent functions (see, e.g., [1-12]).

For two functions $f$ and $g$, analytic in $\mathbb{U}$, we say that the function $f(z)$ is subordinate to $g(z)$ in $\mathbb{U}$, and we write

$$
f(z) \prec g(z) \quad(z \in \mathbb{U}),
$$

if there exists a Schwarz function $w(z)$, analytic in $\mathbb{U}$, with

$$
w(0)=0, \quad|w(z)|<1 \quad(z \in \mathbb{U}),
$$

such that

$$
f(z)=g(w(z)) \quad(z \in \mathbb{U}) .
$$

In particular, if the function $g$ is univalent in $\mathbb{U}$, the above subordination is equivalent to

$$
f(0)=g(0), \quad f(\mathbb{U}) \subset g(\mathbb{U}) .
$$

Let $\varphi(z)$ be an analytic function with

$$
\varphi(0)=1, \quad \varphi^{\prime}(0)>0, \quad \operatorname{Re}\{\varphi(z)\}>0, \quad(z \in \mathbb{U}),
$$

which maps the open unit disk $\mathbb{U}$ onto a star-like region with respect to 1 and is symmetric with respect to the real axis.

This paper contains analogues of (3) for the following classes of analytic functions.

Definition 1. Let

$$
\alpha>0,0 \leq \gamma \leq 1, b \in \mathbb{C} \backslash\{0\} .
$$

A function $f \in \mathscr{A}$ is said to be in the class $\mathscr{R}_{b}(\alpha, \gamma ; \varphi)$ if it satisfies the following subordination condition:

$$
\begin{aligned}
1+\frac{1}{b}\left((1-\alpha+2 \gamma) \frac{f(z)}{z}+(\alpha-2 \gamma) f^{\prime}(z)\right. & \\
\left.+\gamma z f^{\prime \prime}(z)-1\right) & <\varphi(z),
\end{aligned}
$$

where $\varphi(z)$ is defined to be the same as above for $z \in \mathbb{U}$.
Remark 2. (i) If we set

$$
\alpha=2 \gamma+1
$$

in Definition 1, then we have the class

$$
\mathscr{R}_{b}(2 \gamma+1, \gamma ; \varphi)=\mathscr{R}_{\gamma}^{b}(\varphi)
$$

which consists of functions satisfying

$$
1+\frac{1}{b}\left(f^{\prime}(z)+\gamma z f^{\prime \prime}(z)-1\right) \prec \varphi(z) \quad(z \in \mathbb{U}) .
$$

This class was introduced by Bansal [13].

(ii) If we set

$$
\varphi(z)=\frac{1+A z}{1+B z} \quad(-1 \leq B<A \leq 1 ; z \in \mathbb{U})
$$

in Definition 1, then we have a new class

$$
\mathscr{R}_{b}\left(\alpha, \gamma ; \frac{1+A z}{1+B z}\right)=\mathscr{R}_{b}(\alpha, \gamma ; A, B)
$$

which consists of functions satisfying

$$
\begin{gathered}
1+\frac{1}{b}\left((1-\alpha+2 \gamma) \frac{f(z)}{z}+(\alpha-2 \gamma) f^{\prime}(z)\right. \\
\left.+\gamma z f^{\prime \prime}(z)-1\right) \prec \frac{1+A z}{1+B z} .
\end{gathered}
$$

Taking

$$
\alpha=2 \gamma+1
$$

in (25), we have the class

$$
\mathscr{R}_{b}(2 \gamma+1, \gamma ; A, B)=\mathscr{R}_{\gamma}^{b}(A, B)
$$

which consists of functions satisfying

$$
\begin{array}{r}
1+\frac{1}{b}\left(f^{\prime}(z)+\gamma z f^{\prime \prime}(z)-1\right)<\frac{1+A z}{1+B z} \\
(-1 \leq B<A \leq 1 ; z \in \mathbb{U}) .
\end{array}
$$

This class was introduced by Bansal [14].

(iii) If we set

$$
\gamma=0, \quad b=1, \quad A=1, \quad B=-1
$$

in (25), then we have the class

$$
\mathscr{R}_{1}(\alpha, 0 ; 1,-1)=\mathscr{R}(\alpha)
$$

which consists of functions satisfying

$$
\operatorname{Re}\left\{(1-\alpha) \frac{f(z)}{z}+\alpha f^{\prime}(z)\right\}>0 .
$$

This class was introduced by Murugusundaramoorthy and Magesh [15]. The subclass $\mathscr{R}_{1}(1,0 ; 1,-1)=\mathscr{R}(1)=\mathscr{R}$ was studied by MacGregor [16]. 
We denote by $\mathscr{P}$ the class of the analytic functions in $\mathbb{U}$ with

$$
p(0)=1, \quad \operatorname{Re}\{p(z)\}>0 .
$$

We will need the following lemmas.

Lemma 3 (see [12]). If $p \in \mathscr{P}$ with $p(z)=1+c_{1} z+c_{2} z^{2}+\cdots$, then

$$
\left|c_{2}-v c_{1}^{2}\right| \leq \begin{cases}-4 v+2, & v \leq 0 \\ 2, & 0 \leq v \leq 1 \\ 4 v-2, & v \geq 1\end{cases}
$$

When $v<0$ or $v>1$, equality holds true if and only if $p(z)$ is $(1+z) /(1-z)$ or one of its rotations. If $0<v<1$, then equality holds true if and only if $p(z)$ is $\left(1+z^{2}\right) /\left(1-z^{2}\right)$ or one of its rotations. If $v=0$, then the equality holds true if and only if

$$
p(z)=\left(\frac{1}{2}+\frac{1}{2} \eta\right) \frac{1+z}{1-z}+\left(\frac{1}{2}-\frac{1}{2} \eta\right) \frac{1-z}{1+z} \quad(0 \leq \eta \leq 1)
$$

or one of its rotations. If $v=1$, then the equality holds true if and only if $p(z)$ is the reciprocal of one of the functions such that the equality holds true in the case when $v=0$.

Although the above upper bound is sharp, in the case when $0<v<1$, it can be further improved as follows:

$$
\begin{gathered}
\left|c_{2}-v c_{1}^{2}\right|+v\left|c_{1}\right|^{2} \leq 2 \quad\left(0<v \leq \frac{1}{2}\right), \\
\left|c_{2}-v c_{1}^{2}\right|+(1-v)\left|c_{1}\right|^{2} \leq 2 \quad\left(\frac{1}{2}<v \leq 1\right) .
\end{gathered}
$$

Lemma 4 (see [17]). Let $p \in \mathscr{P}$ with $p(z)=1+c_{1} z+c_{2} z^{2}+$ $\cdots$. Then for any complex number $v$

$$
\left|c_{2}-v c_{1}^{2}\right| \leq 2 \max \{1,|2 v-1|\},
$$

and the result is sharp for the functions given by

$$
p(z)=\frac{1+z^{2}}{1-z^{2}}, \quad p(z)=\frac{1+z}{1-z} .
$$

\section{Fekete-Szegö Problem for the Function \\ Class $\mathscr{R}_{b}(\alpha, \gamma ; \varphi)$}

By making use of Lemma 3, we first prove the Fekete-Szegö type inequalities asserted by Theorem 5 below.

Theorem 5. Let

$$
\alpha>0, \quad 0 \leq \gamma \leq 1, \quad b>0 .
$$

Also let

$$
\varphi(z)=1+B_{1} z+B_{2} z^{2}+B_{3} z^{3}+\cdots,
$$

where

$$
B_{1}>0, \quad B_{2} \geq 0 .
$$

If $f(z)$ given by (1) belongs to the function class $\mathscr{R}_{b}(\alpha, \gamma ; \varphi)$, then

$$
\begin{aligned}
& \left|a_{3}-\mu a_{2}^{2}\right| \\
& \leq\left\{\begin{array}{l}
\frac{B_{1} b}{(1+2 \alpha+2 \gamma)}\left(\frac{B_{2}}{B_{1}}-\mu \frac{B_{1} b(1+2 \alpha+2 \gamma)}{(1+\alpha)^{2}}\right), \\
\mu \leq \sigma_{1} \\
\frac{B_{1} b}{(1+2 \alpha+2 \gamma)} \\
\begin{array}{c}
\sigma_{1} \leq \mu \leq \sigma_{2} \\
B_{1} b
\end{array} \\
\frac{(1+2 \alpha+2 \gamma)}{\mu \geq \sigma_{2}}
\end{array}\left(-\frac{B_{2}}{B_{1}}+\mu \frac{B_{1} b(1+2 \alpha+2 \gamma)}{(1+\alpha)^{2}}\right),\right.
\end{aligned}
$$

where

$$
\begin{aligned}
& \sigma_{1}=\frac{\left(B_{2}-B_{1}\right)(1+\alpha)^{2}}{B_{1}^{2} b(1+2 \alpha+2 \gamma)}, \\
& \sigma_{2}=\frac{\left(B_{2}+B_{1}\right)(1+\alpha)^{2}}{B_{1}^{2} b(1+2 \alpha+2 \gamma)}, \\
& \sigma_{3}=\frac{B_{2}(1+\alpha)^{2}}{B_{1}^{2} b(1+2 \alpha+2 \gamma)} .
\end{aligned}
$$

If $\sigma_{1} \leq \mu \leq \sigma_{3}$, then

$$
\begin{aligned}
\mid a_{3}- & \mu a_{2}^{2} \mid+\frac{(1+\alpha)^{2}}{B_{1} b(1+2 \alpha+2 \gamma)} \\
& \times\left[1-\frac{B_{2}}{B_{1}}+\mu \frac{B_{1} b(1+2 \alpha+2 \gamma)}{(1+\alpha)^{2}}\right]\left|a_{2}\right|^{2} \\
\leq & \frac{B_{1} b}{(1+2 \alpha+2 \gamma)} .
\end{aligned}
$$

Furthermore, if $\sigma_{3} \leq \mu \leq \sigma_{2}$, then

$$
\begin{aligned}
\mid a_{3}- & \mu a_{2}^{2} \mid+\frac{(1+\alpha)^{2}}{B_{1} b(1+2 \alpha+2 \gamma)} \\
& \times\left[1+\frac{B_{2}}{B_{1}}-\mu \frac{B_{1} b(1+2 \alpha+2 \gamma)}{(1+\alpha)^{2}}\right]\left|a_{2}\right|^{2} \\
\leq & \frac{B_{1} b}{(1+2 \alpha+2 \gamma)} .
\end{aligned}
$$

Each of these results is sharp.

Proof. Since $f \in \mathscr{R}_{b}(\alpha, \gamma ; \varphi)$, we have

$$
h(z) \prec \varphi(z),
$$

where

$$
\begin{gathered}
h(z)=1+\frac{1}{b}\left((1-\alpha+2 \gamma) \frac{f(z)}{z}+(\alpha-2 \gamma) f^{\prime}(z)\right. \\
\left.+\gamma z f^{\prime \prime}(z)-1\right) \\
=1+h_{1} z+h_{2} z^{2}+\cdots .
\end{gathered}
$$


From (46), we have

$$
h_{1}=\frac{1}{b}(1+\alpha) a_{2}, \quad h_{2}=\frac{1}{b}(1+2 \alpha+2 \gamma) a_{3} .
$$

Since $\varphi(z)$ is univalent and $h(z) \prec \varphi(z)$, the function

$$
p_{1}(z)=\frac{1+\varphi^{-1}(h(z))}{1-\varphi^{-1}(h(z))}=1+c_{1} z+c_{2} z^{2}+c_{3} z^{3}+\cdots
$$

is analytic and has a positive real part in $\mathbb{U}$. Also we have

$$
\begin{aligned}
h(z) & =\varphi\left(\frac{p_{1}(z)-1}{p_{1}(z)+1}\right) \\
& =1+\frac{B_{1} c_{1}}{2} z+\left[\frac{B_{1}}{2}\left(c_{2}-\frac{c_{1}^{2}}{2}\right)+\frac{B_{2} c_{1}^{2}}{4}\right] z^{2}+\cdots .
\end{aligned}
$$

Thus by (47) and (49) we get

$$
\begin{gathered}
a_{2}=\frac{B_{1} c_{1} b}{2(1+\alpha)}, \\
a_{3}=\frac{b}{(1+2 \alpha+2 \gamma)}\left[\frac{B_{1}}{2}\left(c_{2}-\frac{c_{1}^{2}}{2}\right)+\frac{B_{2} c_{1}^{2}}{4}\right] .
\end{gathered}
$$

Taking into account (50), we obtain

$$
\begin{aligned}
a_{3}-\mu a_{2}^{2}= & \frac{b}{(1+2 \alpha+2 \gamma)} \\
& \times\left[\frac{B_{1}}{2}\left(c_{2}-\frac{c_{1}^{2}}{2}\right)+\frac{B_{2} c_{1}^{2}}{4}\right]-\mu \frac{B_{1}^{2} c_{1}^{2} b^{2}}{4(1+\alpha)^{2}} \\
= & \frac{B_{1} b}{2(1+2 \alpha+2 \gamma)}\left(c_{2}-\delta c_{1}^{2}\right)
\end{aligned}
$$

where

$$
\delta=\frac{1}{2}\left(1-\frac{B_{2}}{B_{1}}+\frac{\mu B_{1} b(1+2 \alpha+2 \gamma)}{(1+\alpha)^{2}}\right) .
$$

The assertion of Theorem 5 now follows by an application of Lemma 3. On the other hand, using (51) for the values of $\sigma_{1} \leq$ $\mu \leq \sigma_{3}$, we have

$$
\begin{aligned}
\mid a_{3}- & \left.\mu a_{2}^{2}\left|+\left(\mu-\sigma_{1}\right)\right| a_{2}\right|^{2} \\
= & \frac{B_{1} b}{2(1+2 \alpha+2 \gamma)}\left|c_{2}-\delta c_{1}^{2}\right| \\
& +\left(\mu-\sigma_{1}\right) \frac{B_{1}^{2} b^{2}\left|c_{1}\right|^{2}}{4(1+\alpha)^{2}} \\
= & \frac{B_{1} b}{2(1+2 \alpha+2 \gamma)}\left|c_{2}-\delta c_{1}^{2}\right| \\
& +\left(\mu-\frac{\left(B_{2}-B_{1}\right)(1+\alpha)^{2}}{B_{1}^{2} b(1+2 \alpha+2 \gamma)}\right) \frac{B_{1}^{2} b^{2}\left|c_{1}\right|^{2}}{4(1+\alpha)^{2}} \\
= & \frac{B_{1} b}{2(1+2 \alpha+2 \gamma)}\left\{\left|c_{2}-\delta c_{1}^{2}\right|+\delta\left|c_{1}\right|^{2}\right\} \\
\leq & \frac{B_{1} b}{(1+2 \alpha+2 \gamma)} .
\end{aligned}
$$

Similarly, for the values of $\sigma_{3} \leq \mu \leq \sigma_{2}$, we get

$$
\begin{aligned}
\left|a_{3}-\mu a_{2}^{2}\right|+\left(\sigma_{2}-\mu\right)\left|a_{2}\right|^{2} & \left|c_{1} b-\delta c_{1}^{2}\right| \\
= & \frac{B_{1}^{2} b^{2}\left|c_{1}\right|^{2}}{4(1+\alpha)^{2}} \\
& +\left(\sigma_{2}-\mu\right) \frac{B_{1} b}{2(1+2 \alpha+2 \gamma)}\left|c_{2}-\delta c_{1}^{2}\right| \\
= & +\left(\frac{\left(B_{2}+B_{1}\right)(1+\alpha)^{2}}{B_{1}^{2} b(1+2 \alpha+2 \gamma)}-\mu\right) \frac{B_{1}^{2} b^{2}\left|c_{1}\right|^{2}}{4(1+\alpha)^{2}} \\
= & \frac{B_{1} b}{2(1+2 \alpha+2 \gamma)}\left\{\left|c_{2}-\delta c_{1}^{2}\right|+(1-\delta)\left|c_{1}\right|^{2}\right\} \\
\leq & \frac{B_{1} b}{(1+2 \alpha+2 \gamma)} .
\end{aligned}
$$

To show that the bounds asserted by Theorem 5 are sharp, we define the following functions:

$$
K_{\varphi_{n}}(z) \quad(n=2,3, \ldots),
$$

with

$$
K_{\varphi_{n}}(0)=0=K_{\varphi_{n}}^{\prime}(0)-1
$$

by

$$
\begin{gathered}
1+\frac{1}{b}\left((1-\alpha+2 \gamma) \frac{K_{\varphi_{n}}(z)}{z}+(\alpha-2 \gamma) K_{\varphi_{n}}^{\prime}(z)\right. \\
\left.+\gamma z K_{\varphi_{n}}^{\prime \prime}(z)-1\right)=\varphi\left(z^{n-1}\right),
\end{gathered}
$$


and the functions $F_{\eta}(z)$ and $G_{\eta}(z)(0 \leq \eta \leq 1)$, with

$$
F_{\eta}(0)=0=F_{\eta}^{\prime}(0)-1, \quad G_{\eta}(0)=0=G_{\eta}^{\prime}(0)-1,
$$

by

$$
\begin{array}{r}
1+\frac{1}{b}\left((1-\alpha+2 \gamma) \frac{F_{\eta}(z)}{z}+(\alpha-2 \gamma) F_{\eta}^{\prime}(z)\right. \\
\left.+\gamma z F_{\eta}^{\prime \prime}(z)-1\right)=\varphi\left(\frac{z(z+\eta)}{1+\eta z}\right), \\
1+\frac{1}{b}\left((1-\alpha+2 \gamma) \frac{G_{\eta}(z)}{z}+(\alpha-2 \gamma) G_{\eta}^{\prime}(z)\right. \\
\left.+\gamma z G_{\eta}^{\prime \prime}(z)-1\right)=\varphi\left(-\frac{z(z+\eta)}{1+\eta z}\right),
\end{array}
$$

respectively. Then, clearly, the functions $K_{\varphi_{n}}, F_{\eta}$, and $G_{\eta} \in$ $\mathscr{R}_{b}(\alpha, \gamma ; \varphi)$. We also write

$$
K_{\varphi}=K_{\varphi_{2}}
$$

If $\mu<\sigma_{1}$ or $\mu>\sigma_{2}$, then the equality in Theorem 5 holds true if and only if $f$ is $K_{\varphi}$ or one of its rotations. When $\sigma_{1}<\mu<\sigma_{2}$, then the equality holds true if and only if $f$ is $K_{\varphi_{3}}$ or one of its rotations. If $\mu=\sigma_{1}$, then the equality holds true if and only if $f$ is $F_{\eta}$ or one of its rotations. If $\mu=\sigma_{2}$, then the equality holds true if and only if $f$ is $G_{\eta}$ or one of its rotations.

By making use of Lemma 4, we immediately obtain the following Fekete-Szegö type inequality.

Theorem 6. Let

$$
\alpha>0, \quad 0 \leq \gamma \leq 1, \quad b \in \mathbb{C} \backslash\{0\} .
$$

Also let

$$
\varphi(z)=1+B_{1} z+B_{2} z^{2}+B_{3} z^{3}+\cdots,
$$

where

$$
B_{1}>0, \quad B_{2} \geq 0 .
$$

If $f(z)$ given by (1) belongs to the function class $\mathscr{R}_{b}(\alpha, \gamma ; \varphi)$, then for any complex number $\mu$

$$
\begin{aligned}
& \left|a_{3}-\mu a_{2}^{2}\right| \\
& \quad \leq \frac{B_{1}|b|}{(1+2 \alpha+2 \gamma)} \max \left\{1,\left|\frac{B_{2}}{B_{1}}-\frac{\mu B_{1} b(1+2 \alpha+2 \gamma)}{(1+\alpha)^{2}}\right|\right\} .
\end{aligned}
$$

The result is sharp.

Remark 7. The coefficient bounds for $\left|a_{2}\right|$ and $\left|a_{3}\right|$ are special cases of those asserted by Theorem 5 .

Taking $\alpha=2 \gamma+1$ in Theorem 6 , we have the following corollary.
Corollary 8 (see [13]). Let

$$
0 \leq \gamma \leq 1, \quad b \in \mathbb{C} \backslash\{0\} .
$$

Also let

$$
\varphi(z)=1+B_{1} z+B_{2} z^{2}+B_{3} z^{3}+\cdots,
$$

where

$$
B_{1}>0, \quad B_{2} \geq 0
$$

If $f(z)$ given by (1) belongs to the function class $\mathscr{R}_{\gamma}^{b}(\varphi)$, then for any complex number $\mu$

$$
\begin{aligned}
& \left|a_{3}-\mu a_{2}^{2}\right| \\
& \leq \frac{B_{1}|b|}{3(1+2 \gamma)} \max \left\{1,\left|\frac{B_{2}}{B_{1}}-\frac{3 \mu B_{1} b(1+2 \gamma)}{4(1+\gamma)^{2}}\right|\right\} .
\end{aligned}
$$

The result is sharp.

If we set

$$
\varphi(z)=\frac{1+A z}{1+B z} \quad(-1 \leq B<A \leq 1 ; z \in \mathbb{U})
$$

in Theorem 6, then we have

$$
B_{1}=A-B, \quad B_{2}=-B(A-B) .
$$

So we get the following corollary.

Corollary 9. Let

$$
\alpha>0, \quad 0 \leq \gamma \leq 1, \quad b \in \mathbb{C} \backslash\{0\} .
$$

Also let

$$
\varphi(z)=\frac{1+A z}{1+B z} \quad(-1 \leq B<A \leq 1 ; z \in \mathbb{U}) .
$$

If $f(z)$ given by (1) belongs to the function class $\mathscr{R}_{b}(\alpha, \gamma ; A, B)$, then for any complex number $\mu$

$$
\begin{aligned}
\left|a_{3}-\mu a_{2}^{2}\right| \leq & \frac{(A-B)|b|}{(1+2 \alpha+2 \gamma)} \\
& \times \max \left\{1,\left|B+\frac{\mu(A-B) b(1+2 \alpha+2 \gamma)}{(1+\alpha)^{2}}\right|\right\} .
\end{aligned}
$$

The result is sharp.

Putting $\alpha=2 \gamma+1$ in Corollary 9, we obtain the following corollary.

Corollary 10 (see [13]). Let

$$
0 \leq \gamma \leq 1, \quad b \in \mathbb{C} \backslash\{0\} .
$$

Also let

$$
\varphi(z)=\frac{1+A z}{1+B z} \quad(-1 \leq B<A \leq 1 ; z \in \mathbb{U}) .
$$


If $f(z)$ given by (1) belongs to the function class $\mathscr{R}_{\gamma}^{b}(A, B)$, then for any complex number $\mu$

$$
\begin{aligned}
\left|a_{3}-\mu a_{2}^{2}\right| \leq & \frac{(A-B)|b|}{3(1+2 \gamma)} \\
& \times \max \left\{1,\left|B+\frac{3 \mu(A-B) b(1+2 \gamma)}{4(1+\gamma)^{2}}\right|\right\} .
\end{aligned}
$$

The result is sharp.

Also putting $\gamma=0, b=1, A=1$, and $B=-1$ in Corollary 9 , we obtain the following corollary.

Corollary 11. Let $\alpha>0$. If $f(z)$ given by (1) belongs to the function class $\mathscr{R}(\alpha)$, then for any complex number $\mu$

$$
\left|a_{3}-\mu a_{2}^{2}\right| \leq \frac{2}{(1+2 \alpha)} \max \left\{1,\left|1-\frac{2 \mu(1+2 \alpha)}{(1+\alpha)^{2}}\right|\right\} .
$$

\section{Applications to Analytic Functions Defined by Using Fractional Calculus Operators and Convolution}

The subject of fractional calculus (i.e., calculus of integrals and derivatives of any arbitrary real or complex order) has gained considerable popularity and importance during the past three decades or so. For the applications of the results given in the preceding sections, we first introduce the class $\mathscr{R}_{b}^{\rho}(\alpha, \gamma ; \varphi)$, which is defined by means of the Hadamard product (or convolution) and a certain operator of fractional calculus, known as the Owa-Srivastava operator (see, e.g., $[18,19])$.

Definition 12. The fractional integral of order $\rho$ is defined, for a function $f(z)$, by

$$
D_{z}^{-\rho} f(z)=\frac{1}{\Gamma(\rho)} \frac{d}{d z} \int_{0}^{z} \frac{f(\zeta)}{(z-\zeta)^{1-\rho}} d \zeta \quad(\rho>0)
$$

where the function $f(z)$ is analytic in a simply connected domain of the complex $z$-plane containing the origin, and the multiplicity of $(z-\zeta)^{\rho-1}$ is removed by requiring $\log (z-\zeta)$ to be real when $z-\zeta>0$.

Definition 13. The fractional derivative of $\operatorname{order} \rho$ is defined, for a function $f(z)$, by

$$
D_{z}^{\rho} f(z)=\frac{1}{\Gamma(1-\rho)} \frac{d}{d z} \int_{0}^{z} \frac{f(\zeta)}{(z-\zeta)^{\rho}} d \zeta \quad(0 \leq \rho<1)
$$

where $f(z)$ is constrained, and the multiplicity of $(z-\zeta)^{-\rho}$ is removed, as in Definition 12.
Definition 14. Under the hypotheses of Definition 13, the fractional derivative of order $n+\rho$ is defined, for a function $f(z)$, by

$$
\begin{gathered}
D_{z}^{n+\rho} f(z)=\frac{d^{n}}{d z^{n}}\left(D_{z}^{\rho} f(z)\right), \\
\left(0 \leq \rho<1, n \in \mathbb{N}_{0}=\mathbb{N} \cup\{0\}\right) .
\end{gathered}
$$

Using Definitions 12, 13, and 14, fractional derivatives, and fractional integrals, Owa and Srivastava [20] introduced the operator $\Omega^{\rho}: \mathscr{A} \rightarrow \mathscr{A}$ defined by

$$
\begin{aligned}
\left(\Omega^{\rho} f\right)(z) & =\Gamma(2-\rho) z^{\rho} D_{z}^{\rho} f(z), \quad \rho \neq 2,3,4, \ldots \\
& =z+\sum_{n=2}^{\infty} \frac{\Gamma(n+1) \Gamma(2-\rho)}{\Gamma(n-\rho+1)} a_{n} z^{n} .
\end{aligned}
$$

This operator is known as the Owa-Srivastava operator. In terms of the Owa-Srivastava operator $\Omega^{\rho}$ defined by (81), we now introduce the function class $\mathscr{R}_{b}^{\rho}(\alpha, \gamma ; \varphi)$ in the following way:

$$
\mathscr{R}_{b}^{\rho}(\alpha, \gamma ; \varphi)=\left\{f \in \mathscr{A}: \Omega^{\rho} f \in \mathscr{R}_{b}(\alpha, \gamma ; \varphi)\right\} .
$$

Note that the function class $\mathscr{R}_{b}^{\rho}(\alpha, \gamma ; \varphi)$ is a special case of the function class $\mathscr{R}_{b}^{g}(\alpha, \gamma ; \varphi)$ when

$$
g(z)=z+\sum_{n=2}^{\infty} \frac{\Gamma(n+1) \Gamma(2-\rho)}{\Gamma(n-\rho+1)} z^{n} .
$$

Now suppose that

$$
g(z)=z+\sum_{n=2}^{\infty} g_{n} z^{n} \quad\left(g_{n}>0\right)
$$

Since

$$
\begin{aligned}
& f(z)=z+\sum_{n=2}^{\infty} a_{n} z^{n} \in \mathscr{R}_{b}^{g}(\alpha, \gamma ; \varphi) \\
& \Longleftrightarrow(f * g)(z)=z+\sum_{n=2}^{\infty} g_{n} a_{n} z^{n} \in \mathscr{R}_{b}(\alpha, \gamma ; \varphi),
\end{aligned}
$$

we can obtain the coefficient estimates for functions in the class $\mathscr{R}_{b}^{g}(\alpha, \gamma ; \varphi)$ from the corresponding estimates for functions in the class $\mathscr{R}_{b}(\alpha, \gamma ; \varphi)$. By applying Theorem 5 to the following Hadamard product (or convolution):

$$
(f * g)(z)=z+g_{2} a_{2} z^{2}+g_{3} a_{3} z^{3}+\cdots,
$$

we get the following theorem after an obvious change of the parameter $\mu$.

Theorem 15. Let

$$
\alpha>0, \quad 0 \leq \gamma \leq 1, \quad b>0 .
$$

Also let

$$
\varphi(z)=1+B_{1} z+B_{2} z^{2}+B_{3} z^{3}+\cdots,
$$


where

$$
B_{1}>0, \quad B_{2} \geq 0, \quad g_{n}>0 \quad(n=3,4, \ldots) .
$$

If $f(z)$ given by (1) belongs to the function class $\mathscr{R}_{b}^{g}(\alpha, \gamma ; \varphi)$, then

$$
\begin{aligned}
& \left|a_{3}-\mu a_{2}^{2}\right| \\
& \leq\left\{\begin{array}{l}
\frac{B_{1} b}{(1+2 \alpha+2 \gamma) g_{3}}\left(\frac{B_{2}}{B_{1}}-\mu \frac{g_{3}}{g_{2}^{2}} \frac{B_{1} b(1+2 \alpha+2 \gamma)}{(1+\alpha)^{2}}\right), \\
\mu \leq \sigma_{1} \\
\frac{B_{1} b}{(1+2 \alpha+2 \gamma) g_{3}}, \\
\sigma_{1} \leq \mu \leq \sigma_{2} \\
\frac{B_{1} b}{(1+2 \alpha+2 \gamma) g_{3}}\left(-\frac{B_{2}}{B_{1}}+\mu \frac{g_{3}}{g_{2}^{2}} \frac{B_{1} b(1+2 \alpha+2 \gamma)}{(1+\alpha)^{2}}\right), \\
\mu \geq \sigma_{2},
\end{array}\right.
\end{aligned}
$$

where

$$
\begin{gathered}
\sigma_{1}=\frac{g_{2}^{2}}{g_{3}} \frac{\left(B_{2}-B_{1}\right)(1+\alpha)^{2}}{B_{1}^{2} b(1+2 \alpha+2 \gamma)}, \\
\sigma_{2}=\frac{g_{2}^{2}}{g_{3}} \frac{\left(B_{2}+B_{1}\right)(1+\alpha)^{2}}{B_{1}^{2} b(1+2 \alpha+2 \gamma)} .
\end{gathered}
$$

Each of these results is sharp.

When $g$ corresponds to the Owa-Srivastava operator given in (82), we obtain

$$
\begin{gathered}
g_{2}=\frac{\Gamma(3) \Gamma(2-\rho)}{\Gamma(3-\rho)}=\frac{2}{2-\rho}, \\
g_{3}=\frac{\Gamma(4) \Gamma(2-\rho)}{\Gamma(4-\rho)}=\frac{6}{(2-\rho)(3-\rho)} .
\end{gathered}
$$

For $g_{2}$ and $g_{3}$ given by (93) and (94), respectively, Theorem 15 reduces to the following result.

Theorem 16. Let

$$
\alpha>0, \quad 0 \leq \gamma \leq 1, \quad b>0 .
$$

Also let

$$
\varphi(z)=1+B_{1} z+B_{2} z^{2}+B_{3} z^{3}+\cdots,
$$

where

$$
B_{1}>0, \quad B_{2} \geq 0 .
$$

If $f(z)$ given by (1) belongs to the function class $\mathscr{R}_{b}^{g}(\alpha, \gamma ; \varphi)$, then

$$
\left|a_{3}-\mu a_{2}^{2}\right|
$$

$$
\leq\left\{\begin{array}{l}
\frac{(2-\rho)(3-\rho) B_{1} b}{6(1+2 \alpha+2 \gamma)}\left(\frac{B_{2}}{B_{1}}-\mu \frac{3(2-\rho)}{2(3-\rho)} \frac{B_{1} b(1+2 \alpha+2 \gamma)}{(1+\alpha)^{2}}\right), \\
\mu \leq \sigma_{1} \\
\frac{(2-\rho)(3-\rho) B_{1} b}{6(1+2 \alpha+2 \gamma)}, \\
\sigma_{1} \leq \mu \leq \sigma_{2} \\
\frac{(2-\rho)(3-\rho) B_{1} b}{6(1+2 \alpha+2 \gamma)}\left(-\frac{B_{2}}{B_{1}}+\mu \frac{3(2-\rho)}{2(3-\rho)} \frac{B_{1} b(1+2 \alpha+2 \gamma)}{(1+\alpha)^{2}}\right), \\
\mu \geq \sigma_{2}
\end{array}\right.
$$

where

$$
\begin{aligned}
& \sigma_{1}=\frac{2(3-\rho)}{3(2-\rho)} \frac{\left(B_{2}-B_{1}\right)(1+\alpha)^{2}}{B_{1}^{2} b(1+2 \alpha+2 \gamma)}, \\
& \sigma_{2}=\frac{2(3-\rho)}{3(2-\rho)} \frac{\left(B_{2}+B_{1}\right)(1+\alpha)^{2}}{B_{1}^{2} b(1+2 \alpha+2 \gamma)} .
\end{aligned}
$$

Each of these results is sharp.

\section{Conflict of Interests}

The author declares that there is no conflict of interests regarding the publication of this paper.

\section{References}

[1] M. Fekete and G. Szegö, "Eine bemerkung über ungerade schlichte funktionen," Journal of the London Mathematical Society, vol. 8, pp. 85-89, 1933.

[2] H. R. Abdel-Gawad and D. K. Thomas, "The Fekete-Szegö problem for strongly close-to-convex functions," Proceedings of the American Mathematical Society, vol. 114, no. 2, pp. 345-349, 1992.

[3] H. S. Al-Amiri, "Certain generalizations of prestarlike functions," Australian Mathematical Society A, vol. 28, no. 3, pp. 325334,1979

[4] J. H. Choi, Y. C. Kim, and T. Sugawa, "A general approach to the Fekete-Szegö problem," Journal of the Mathematical Society of Japan, vol. 59, no. 3, pp. 707-727, 2007.

[5] A. Chonweerayoot, D. K. Thomas, and W. Upakarnitikaset, "On the Fekete-Szegö theorem for close-to-convex functions," Institut Mathématique, vol. 66, pp. 18-26, 1992.

[6] M. Darus and D. K. Thomas, "On the Fekete-Szegö theorem for close-to-convex functions," Mathematica Japonica, vol. 44, no. 3, pp. 507-511, 1996.

[7] M. Darus and D. K. Thomas, "On the Fekete-Szegö theorem for close-to-convex functions," Mathematica Japonica, vol. 47, no. 1, pp. 125-132, 1998.

[8] S. Kanas and A. Lecko, "On the Fekete-Szegö problem and the domain of convexity for a certain class of univalent functions," Folia Scientiarum, Universitatis Technicae Resoviensis, no. 10, pp. 49-57, 1990. 
[9] F. R. Keogh and E. P. Merkes, "A coefficient inequality for certain classes of analytic functions," Proceedings of the American Mathematical Society, vol. 20, pp. 8-12, 1969.

[10] W. Koepf, "On the Fekete-Szegö problem for close-to-convex functions," Proceedings of the American Mathematical Society, vol. 101, no. 1, pp. 89-95, 1987.

[11] R. R. London, "Fekete-Szegö inequalities for close-to-convex functions," Proceedings of the American Mathematical Society, vol. 117, no. 4, pp. 947-950, 1993.

[12] W. C. Ma and D. Minda, "A unified treatment of some special classes of univalent functions," in Proceedings of the Conference on Complex Analysis (Tianjin, 1992), Conference on Proceedings Lecture Notes for Analysis, I, pp. 157-169, International Press, Cambridge, Mass, USA, 1994.

[13] D. Bansal, "Fekete-Szegö problem for a new class of analytic functions," International Journal of Mathematics and Mathematical Sciences, vol. 2011, Article ID 143095, 5 pages, 2011.

[14] D. Bansal, "Upper bound of second Hankel determinant for a new class of analytic functions," Applied Mathematics Letters, vol. 26, no. 1, pp. 103-107, 2013.

[15] G. Murugusundaramoorthy and N. Magesh, "Coefficient inequalities for certain classes of analytic functions associated with Hankel determinant," Bulletin of Mathematical Analysis and Applications, vol. 1, no. 3, pp. 85-89, 2009.

[16] T. H. MacGregor, "Functions whose derivative has a positive real part," Transactions of the American Mathematical Society, vol. 104, pp. 532-537, 1962.

[17] V. Ravichandran, A. Gangadharan, and M. Darus, "FeketeSzegö inequality for certain class of Bazilevic functions," Far East Journal of Mathematical Sciences (FJMS), vol. 15, no. 2, pp. 171-180, 2004.

[18] S. Owa, "On the distortion theorems. I," Kyungpook Mathematical Journal, vol. 18, no. 1, pp. 53-59, 1978.

[19] H. M. Srivastava and S. Owa, Univalent Functions, Fractional Calculus, and Their Applications, Halsted Press (Ellis Horwood Limited, Chichester); John Wiley and Sons, New York, NY, USA, 1989.

[20] S. Owa and H. M. Srivastava, "Univalent and starlike generalized hypergeometric functions," Canadian Journal of Mathematics, vol. 39, no. 5, pp. 1057-1077, 1987. 


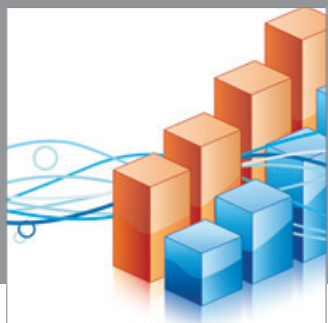

Advances in

Operations Research

mansans

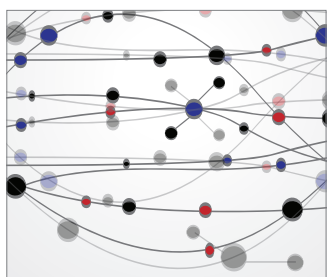

The Scientific World Journal
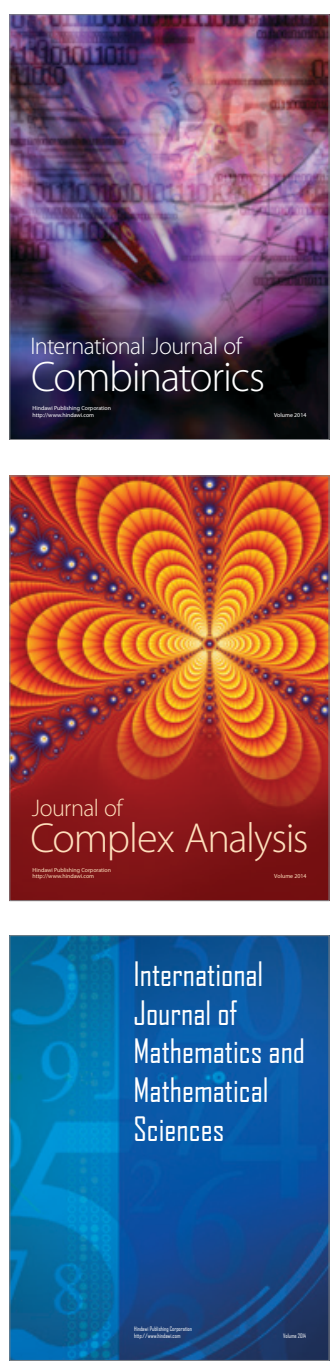
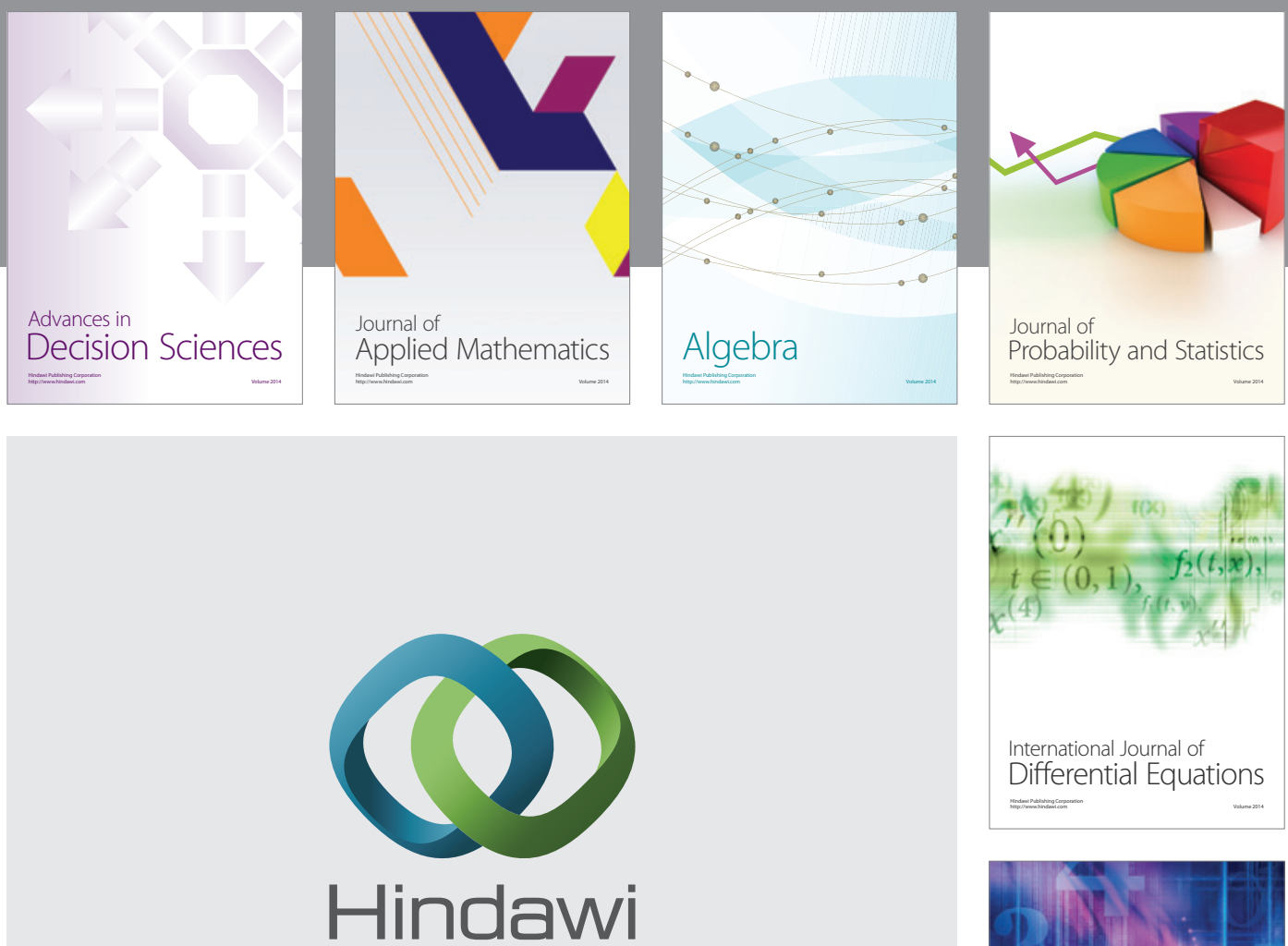

Submit your manuscripts at http://www.hindawi.com
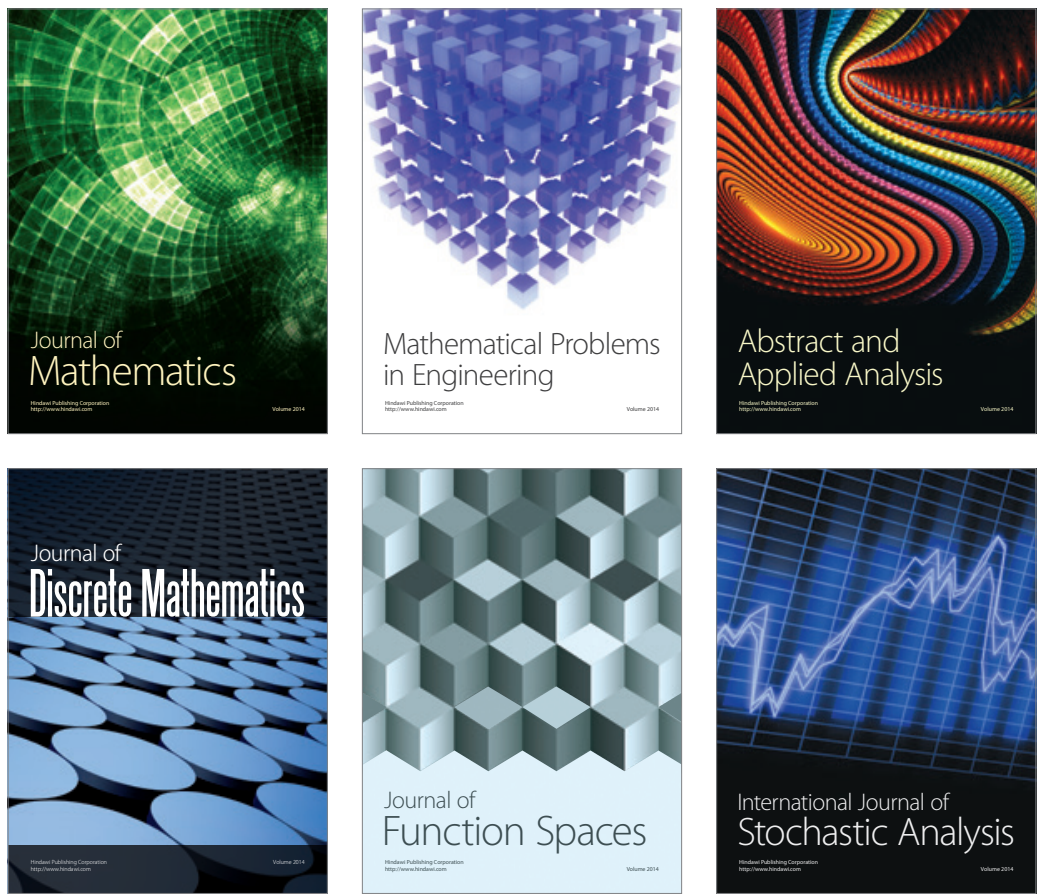

Journal of

Function Spaces

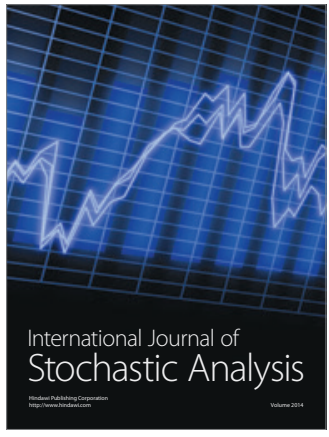

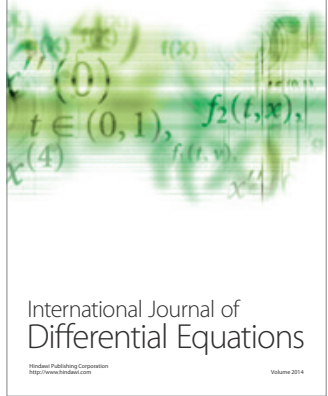
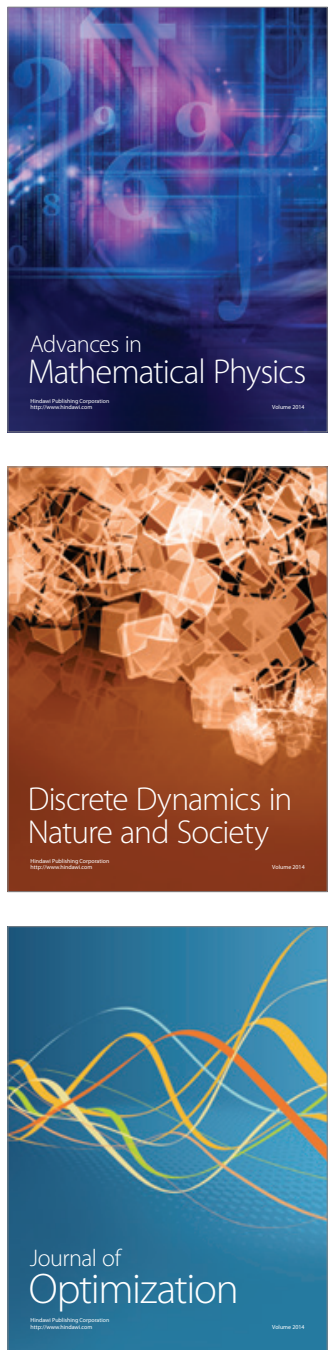